\title{
Complexity in Neurology and Psychiatry
}

\author{
H. A. Braun · F. Moss • S. Postnova • E. Mosekilde
}

Published online: 6 November 2008

(C) Springer Science + Business Media B.V. 2008

This special issue focuses on the most complex system we know: the brain. More precisely, it deals with the examination of the brain dynamics and disturbances which are clinically manifested in neurological and psychiatric disorders like epilepsy, Parkinson's disease or mental depression. These diseases are often associated with disturbances of autonomous functions like hormone secretion and sleep which also are under the control of the brain. Accordingly, the papers in this issue will refer to a diversity of physiological systems. Their common link is that they all are related to brain functions and dysfunctions and that these neurophysiological issues are addressed by physically based approaches of systems analysis with the use of computer models and tools for nonlinear data analysis.

The high expectations in such interdisciplinary approaches towards a better understanding of brain dynamics is the major motivation for this issue. All the authors have expertise in interdisciplinary work and have already made essential contributions in their fields. Many of them are experimental physiologists or clinicians who have learnt to implement biophysical methods, mostly in cooperation with physicists and mathematicians. Vice versa, the physicists and mathematicians among the authors have successfully entered the life sciences in demonstrating that physical applications are not only of interest from a systems theoretical point of view; but can also have high physiological relevance.

H. A. Braun $(\varangle) \cdot$ S. Postnova

Laboratory of Neurodynamics, Institute of Physiology, Philipps University of Marburg,

Deutschhaustr. 2, 35037 Marburg, Germany

e-mail: braun@staff.uni-marburg.de

S. Postnova

e-mail: postnova@staff.uni-marburg.de

F. Moss

Center for Neurodynamics, Department of Physics and Astronomy, University of Missouri Saint Louis, One University Boulevard St. Louis MO 63121, Saint Louis, USA

e-mail: MossF@msx.umsl.edu

E. Mosekilde

Department of Physics, Technical University of Denmark, Fysikvij 309, 2800 Lyngby, Denmark e-mail: Erik.Mosekilde@fysik.dtu.dk 
The authors were participants in the interdisciplinary workshop "From Complex System Theory to Clinical Neurology" which was held at the Max-Planck-Institute for the Physics of Complex Systems (MPIPKS) in Dresden, Germany from June 4 to 8, 2007. This workshop was organised by the guest editors of this issue and has received significant support by the MPIPKS guest programme, not only financial but also including the local organisation which was overtaken by Mandy Lochar and Renate Seidel under the auspices of the MPIPKS Director Frank Jülicher.

Additional support for this workshop was received from the European Commission via "BioSim", a "Network of Excellence" in biosimulation. The goal of this network is to develop modelling and simulation approaches that can lead to a better understanding of physiological processes and which also can help the pharmaceutical industry in its search for more rational strategies of drug development and testing. Computer simulations of physiological processes and drug effects can lead to a more precise, goal-directed design of animal experiments and clinical studies for drug testing. System and data analysis tools can provide further insights into physiologically relevant dynamics and their alteration by a specific drug. This EU project has attracted much attention and is considered as one of the most successful and productive Networks of Excellence in the 6th Framework Programme. It has brought together theoreticians, experimentalists and clinicians for common work on complex systems with practical applications for the treatment of disease.

Despite the remarkable success of the "BioSim" project, applications of nonlinear dynamics tools in biological, physiological and especially in medically relevant clinical research are still the exception rather than the rule. In the neurosciences, indeed, the contributions from nonlinear systems theory are clearly increasing since several years, which is also indicated by an increasing number of computational neuroscience meetings and specialised journals. However, different groups, for example computational neuroscientists, biophysicists and experimental or clinical neurobiologists, are mostly still going their own ways without much exchange, as evidenced by their specialised meetings. Real interdisciplinary work, although prominently emphasised, has only been fully established in and between comparatively few research groups.

It was the motivation of the MPIPKS workshop to bridge the gap between theoreticians of nonlinear dynamics on the one side and clinical researchers on the other side. It is the intention of this issue of the Journal of Biological Physics, titled "Complexity in Neurology and Psychiatry", to attract more open-minded scientists from different fields for clinically relevant, interdisciplinary research.

All papers in this special issue deal with applications of physically based methods of nonlinear systems theory to clinically relevant questions of complex brain dynamics. Some of the papers address general questions of physiologically appropriate modelling approaches and data analysis procedures. Others refer to specific diseases or dysfunctions at various levels. It is particularly interesting to see that complex phenomena in psychiatry and neurology can successfully be modelled on quite different levels of system reduction.

We open the special issue with two review articles. Lehnertz [1] focuses on the development and prevention of epileptic seizures on the basis of appropriate electroencephalogram (EEG) analysis with methods from nonlinear systems theory. Then, Sannita [2] raises general conceptual questions about the brain's robustness and flexibility from a clinician's point of view, specifically emphasising collective behaviours of functionally diverse systems.

Next, a promising and extremely reduced approach is described by Rulkov and Bazhenov [3] who have developed map-based models for large-scale simulations, which nevertheless 
allow us to consider synaptic interconnections and intrinsic neural states in order to capture the main response properties of cortical network dynamics. Large-scale simulations, with a different type of dimension reduction, are also used by Tukhlina and Rosenblum [4] for the development of efficient techniques for manipulation of pathological brain rhythms.

Indeed, from a clinical point of view, brain rhythms, which are built up from neuronal oscillations with different degrees of synchronisation, are among the most important issues of neuronal dysfunction. The development of clinically applicable control stimuli first of all requires reliable detection of the transitions from a physiological to a pathophysiological state. Particularly sophisticated methods have been developed by Martínez-Montes et al. [5] on the basis of combined EEG and functional magnetic resonance imaging recordings for spatiotemporal characterisation of brain networks. Asai and Villa [6] describe a new method for the detection of recurrent temporal patterns in noisy spike trains as a robust expression of the underlying dynamics. The linear autoregressive models of Olbrich and Achermann [7] are specifically developed for the analysis of the temporal organisation of sleep spindles and slow waves in the EEG. These papers show that the EEG, despite a myriad of recently introduced brain imaging techniques, still belongs among the most important methods in clinical practice for the diagnosis of neurological and psychiatric disorders.

The following two papers deal with Parkinson's disease. Modolo et al. [8] compare the outcomes of a simple firing rate model and a population-based model of the subthalamopallidal complex for a better understanding and improved treatment of Parkinson's tremor. Popovych et al. [9] propose nonlinear delayed feedback stimulations for the control of collective dynamics with specific regard to the prevention and interruption of the abnormal synchrony in clinical practice.

However, synchronisation is not necessarily detrimental to physiological functions but can also be required for normal brain function, e.g. for smooth visual pursuit of a moving target. Contreras et al. [10] have used the nonlinear dynamical technique of stochastic phase synchronisation to compare fatigue effects on eye-target synchronisation in both healthy and brain-injured subjects.

Multiple meshing of different functions is also a major issue of several other reports, especially when this complexity is related to the control of autonomous functions. Disturbances of homeostatic, autonomous functions like sleep and hormone secretion are often closely associated with neurological and especially psychiatric diseases. Homeostatic parameters can therefore be used as biological markers. An example is given by Figueroa Helland et al. [11] who describe new algorithms for the analysis of sleep EEG to evaluate drug effects in patients with major depression. The interdependence between the central and autonomic nervous system is particularly emphasised by Pereda and González [12], who have analysed central nervous system (CNS) signals (EEG) in parallel with circulation and respiration parameters in search of clinically useable biological markers, e.g. for the prevention of sudden infant death.

Interdependences between the CNS and autonomous functions are also the focus of Langemann and Peters [13] who present a simple mathematical model of appetite regulation and energy transport from the body into the brain. The development of cyclic food intake is simulated by the interaction between neurons with appetite-amplifying and reducing neuropeptides. The work of Pedersen and Sørensen [14] is also related to energy control, as it demonstrates that limited coordination of spatial waves in insulin-secreting pancreatic $\beta$-cells in the case of spatial variations of glucose concentration can be simulated by means of a perturbed Fisher's equation. 
The last two papers in this issue address specific questions of cellular interactions which nevertheless are of rather general interest. The simulations of Hutt and SchimanskyGeier [15] are designed for the evaluation of the effects of anaesthetics such as propofol. Their neuronal population model includes excitatory and inhibitory synapses and thereby exhibits a bifurcation structure which can be related to the transitions from consciousness to non-consciousness. Finally, the mechanism-based model developed by Postnov et al. [16] considers, in addition to conventional synapses, the impact of glial cells, which is still underestimated, and in most neuronal models even completely neglected, although its functional significance is well demonstrated by a manifold of experimental data.

We expect that the papers of this special issue convincingly demonstrate the value of physically based approaches to the analysis of neuronal dynamics and their disturbances. We hope that this contributes to a broader acceptance of such methods among experimental physiologists and clinicians. We specifically hope that it can inspire more physicists, mathematicians and computer scientists to examine the "Complexity in Neurology and Psychiatry".

Acknowledgements We thank the MPIPKS Dresden and the BioSim EU Network of Excellence (No. LSHB-CT-2004-005137) for generous support of our interdisciplinary activities.

\section{References}

1. Lehnertz, K.: Epilepsy and nonlinear dynamics. J. Biol. Phys. 34(3-4), 253-266 (2008)

2. Sannita, W.: Neuronal functional diversity and collective behaviors. J. Biol. Phys. 34(3-4), 267-278 (2008)

3. Rulkov, N.F., Bazhenov, M.: Oscillations and synchrony in large-scale cortical network models. J. Biol. Phys. 34(3-4), 279-299 (2008)

4. Tukhlina N., Rosenblum M.: Feedback suppression of neuronal synchrony in two interacting populations by vanishing stimulation. J. Biol. Phys. 34(3-4), 301-314 (2008)

5. Martínez-Montes, E., Vega-Hernández, M., Sánchez-Bornot, J.M., Valdés-Sosa, P.A.: Identifying complex brain networks using penalized regression methods. J. Biol. Phys. 34(3-4), 315-323 (2008)

6. Asai, Y., Villa, A.E.P.: Reconstruction of underlying nonlinear deterministic dynamics embedded in noisy spike trains. J. Biol. Phys. 34(3-4), 325-340 (2008)

7. Olbrich, E., Achermann, P.: Analysis of the temporal organization of sleep spindles in the human sleep EEG using a phenomenological modeling approach. J. Biol. Phys. 34(3-4), 341-349 (2008)

8. Modolo, J., Henry, J., Beuter, A.: Dynamics of the subthalamo-pallidal complex in Parkinson's disease during deep brain stimulation. J. Biol. Phys. 34(3-4), 351-366 (2008)

9. Popovych, O.V., Hauptmann, C., Tass, P.A.: Impact of nonlinear delayed feedback on synchronized oscillators. J. Biol. Phys. 34(3-4), 367-379 (2008)

10. Contreras, R., Kolster, R., Voss, H.U., Ghajar, J., Suh, M., Bahar, S.: Eye-target synchronization in mild traumatic brain injured patients. J. Biol. Phys. 34(3-4), 381-392 (2008)

11. Figueroa Helland, V.C., Postnova, S., Schwarz, U., Kurths, J., Kundermann, B., Hemmeter, U., Braun, H.A.: Comparison of different methods for the evaluation of treatment effects from the sleep EEG of patients with major depression. J. Biol. Phys. 34(3-4), 393-404 (2008)

12. Pereda, E., González, J.J.: Nonlinear dynamical analysis of the interdependence between central and autonomic nervous systems in neonates during sleep. J. Biol. Phys. 34(3-4), 405-412 (2008)

13. Langemann, D., Peters, A. Deductive functional assignment of elements in appetite regulation. J. Biol. Phys. 34(3-4), 413-424 (2008)

14. Pedersen, M.G., Sørensen, M.P.: Wave-block due to a threshold gradient underlies limited coordination in pancreatic islets. J. Biol. Phys. 34(3-4), 425-432 (2008)

15. Hutt, A., Schimansky-Geier, L.: Anesthetic-induced transitions by propofol modeled by nonlocal neural populations involving two neuron types. Biol. Phys. 34(3-4), 433-440 (2008)

16. Postnov, D.E., Ryazanova, L.S., Brazhe, N.A., Brazhe, A.R., Maximov, G.V., Mosekilde, E., Sosnovtseva, O.V.: Giant glial cell: new insight through mechanism-based modelling. Biol. Phys. 34(3-4), 441-457 (2008) 